\title{
Utilização de casca de ovo como fonte alternativa de cálcio na obtenção de titanato de cálcio $\left(\mathrm{CaTiO}_{3}\right)$
}

\author{
Use of eggshell as an alternative source of calcium \\ to obtain calcium titanate (CaTiO3)
}

Guilherme Gralik ${ }^{1}$, Giovany Biava ${ }^{1}$

\author{
${ }^{1}$ Serviço Nacional De Aprendizagem Industrial, SENAI, Rua Joaquim de Paula Xavier, 1050, Ponta Grossa, Paraná, \\ Brasil. \\ e-mail: guilherme_gralik@yahoo.com.br, giovany.biava@sistemafiep.org.br
}

\section{RESUMO}

No presente trabalho, detalha-se o estudo do reaproveitamento da casca de ovo como fonte alternativa mineral de calcita na síntese de titanato de cálcio $\left(\mathrm{CaTiO}_{3}\right)$. As matérias-primas foram caracterizadas por difração e fluorescência de raios X e as composições foram formuladas e sinterizadas em diferentes condições. Após a sinterização, as fases cristalinas formadas foram quantificadas pelo método Rietveld (MR), análises de densidade aparente (DA), espectroscopia de impedância (EIA) e de microscopia eletrônica de varrredura (MEV) foram realizadas. Os resultados revelam a possibilidade de se utilizar resíduo de casca de ovo como fonte de cálcio para a formação de titanato de cálcio com estrutura perovskita. É fundamental que estudos de viabilidade técnico-econômica sejam também desenvolvidos para que possíveis aplicações de interesse sejam viáveis.

Palavras-chave: Casca de ovo. Perovskita. Resíduo. Titanato de Cálcio.

\begin{abstract}
We describe the study of the re-utilization of egg shell as an alternative mineral source of calcite in the synthesis of calcium titanate $\left(\mathrm{CaTiO}_{3}\right)$. The raw materials were characterized by $\mathrm{X}$-ray diffraction and $\mathrm{X}$-ray fluorescence; compositions were formulated and sintered under different conditions. After sintering the crystalline phases formed were quantified by the Rietvield (MR) method, analyzes of apparent density (DA), impedance spectroscopy (EIA) and scanning electron microscopy (SEM) were performed. The results reveal the possibility of using the egg shell residue as a source of calcium for the formation of calcium titanate with perovskite structure, it is fundamental to consider studies of technical and economic viability are also developed so that possible applications of interest are feasible.
\end{abstract}

Keywords: Calcium titanate. Eggshell. Perovskite. Waste.

\section{INTRODUÇÃO}

Entre suas várias prerrogativas, a Política Nacional de Resíduos Sólidos (PNRS) (Lei no 12.305/2010) apoia a separação dos resíduos domiciliares e a coleta seletiva de resíduos sólidos realizada de porta em porta [1]. Um dos resíduos gerados nos domicílios é a casca de ovo, que representa, em média, 10\% do peso do ovo, gerando uma quantidade de resíduos de 5,92 milhões de toneladas por ano no mundo inteiro [2]. A valorização desse resíduo sólido vem sendo estudada em várias aplicações: como matéria-prima na síntese de biomateriais [2], em formulações de cerâmica de revestimento [3] e como corretivo alcalinizante em solo [4].

Uma possível valorização desse resíduo é ser utilizado como fonte de cálcio na síntese de titanato de cálcio $\left(\mathrm{CaTiO}_{3}\right)$, que é um material de estrutura perovskita do tipo $\mathrm{ABX} 3$ [5] e não apresenta anisotropia. Sua estrutura é cúbica, acima de $1.307^{\circ} \mathrm{C}$, tetragonal, entre $1.107^{\circ} \mathrm{C}$ e $1.227^{\circ} \mathrm{C}$, e ortorrômbica, abaixo de $1.107^{\circ} \mathrm{C}$ $[6,7]$. A estrutura cúbica pode ser obtida na temperatura ambiente quando o titanato de cálcio $\left(\mathrm{CaTiO}_{3}\right)$ é dopado com cátions receptores $[6,8]$. 
Por suas excelentes propriedades elétricas e magnéticas, a estrutura da perovskita é amplamente utilizada em sensores e dispositivos eletrônicos [9-11]. A estrutura da perovskita $\mathrm{ABX} 3$ é representada como um cubo com os cátions A e B localizados em seus vértices e o ânion X localizado em seus centros da face [12].

$\mathrm{O}$ titanato de cálcio $\left(\mathrm{CaTiO}_{3}\right)$ é um material muito interessante para áreas como geologia, metalurgia e cerâmica, uma vez que é promissor para ser usado em sensores e dispositivos eletrônicos [13]. Também já foram desenvolvidas outras aplicações, como biomaterial usado para revestir próteses de titânio [14] e como elemento de resistência com sensibilidade térmica, em razão de seu coeficiente de temperatura negativo (NTC)[15]. Outra aplicação recente é sua utilização para separar hidrogênio e oxigênio da água destilada durante a conversão de energia luminosa. As propriedades fotoluminescentes do titanato de cálcio $\left(\mathrm{CaTiO}_{3}\right)$ estruturalmente desordenadas também são relatadas na literatura [16,17].

$\mathrm{O}$ titanato de cálcio $\left(\mathrm{CaTiO}_{3}\right)$ apresenta condução iônica e eletrônica em baixas pressões parciais de oxigênio e mantém essa propriedade com altas concentrações de defeitos [18]. Tais defeitos a altas temperaturas podem ser descritos como vacâncias de oxigênio e excesso de elétrons, sendo a condutividade elétrica proporcional à concentração desses defeitos de vacância de oxigênio [19,20]. O titanato de cálcio dopado com diferentes elementos permite obter compostos com propriedades elétricas interessantes situados em uma ampla gama de frequências e temperaturas [21,22].

A técnica de espectroscopia de impedância tem sido aplicada como um dos instrumentos mais versáteis e sensíveis no estudo das propriedades específicas dos materiais, como propriedades elétricas, dielétricas, caracterização de defeitos e transições de fases. Em geral, a modelagem dos dados de impedância é feita por meio de circuitos elétricos equivalentes, os quais descrevem os processos de polarização que ocorrem no material. As componentes real e imaginária são representadas no diagrama de impedância ou de Nyquist, que consiste na representação da parte imaginária da impedância em função da parte real, para cada frequência medida [23,24].

Desta forma, neste trabalho, buscou-se avaliar a formação de titanato de cálcio $\left(\mathrm{CaTiO}_{3}\right)$, com estrutura perovskita, a partir de uma fonte alternativa de cálcio (casca de ovo), analisando suas características microestruturais e a resistividade elétrica.

\section{MATERIAIS E MÉTODOS}

Cascas de ovos foram devidamente lavadas com água e álcool etílico e, posteriormente, secas em estufa com circulação de ar a $110^{\circ} \mathrm{C}$ por 24 horas. O dióxido de titânio (cristal do Brasil) utilizado é um material comercial, com alto teor de pureza $\left(\mathrm{TiO}_{2}: 99 \%\right)$.

As matérias-primas foram moídas separadamente, em moinho planetário, por duas horas a seco, em um jarro de porcelana (um terço de esferas de alumina, um terço de matéria-prima e um terço vazio, em volume). Nas amostras dos pós-resultantes do processo de moagem, foram realizadas análises de fluorescência (FRX) e difração de raios X (DRX). As difrações de raios X (Shimadzu XDR 6000) foram realizadas com amostras em pó (200 Mesh), a uma velocidade de $2 \%$ minuto e em um intervalo de varrimento de 15 a $80^{\circ}$. Os difratogramas de raios $\mathrm{x}$ (DRX) foram identificados utilizando-se a base de um programa comercial (Match Phase Identification from Powder Difraction, versão 1.11).

Com o objetivo de avaliar a formação de titanato de cálcio $\left(\mathrm{CaTiO}_{3}\right)$, as composições foram formuladas e sinterizadas conforme descrito na Tabela 1 .

Tabela 1: Composições formuladas e condições de processamento utilizadas.

\begin{tabular}{l|l|l|l|l|l}
\hline AMOSTRA & $\mathrm{TiO}_{2}(\mathbf{g})$ & $\begin{array}{l}\mathrm{CaCO}_{3}(\mathbf{g}) \\
(\mathrm{CASCA} \text { DE OVO) }\end{array}$ & $\begin{array}{l}\text { RELAÇÃO MOLAR } \\
(\mathrm{Ca}: \mathrm{Ti})\end{array}$ & $\begin{array}{l}\text { TEMPERATURA DE } \\
\text { SINTERIZAÇÃO }\left({ }^{\circ} \mathbf{C}\right)\end{array}$ & PATAMAR (H) \\
\hline $\mathrm{A} 1$ & 10 & 8 & 1,4 & 1200 & 3 \\
\hline $\mathrm{A} 2$ & 10 & 8 & 1,4 & 1200 & 13 \\
\hline $\mathrm{A} 3$ & 10 & 8 & 1,4 & 1300 & 2 \\
\hline
\end{tabular}

Após moagem, as matérias-primas foram pesadas em uma balança analítica (AX200 Shimadzu) e homogeneizadas manualmente, em gral e pistilo em álcool etílico, por cinco minutos. Na sequência, as misturas de pós cerâmicos foram conformadas por prensagem em uma matriz metálica com 100 MPa. Pastilhas com $10 \mathrm{~mm}$ de diâmetro e aproximadamente $2 \mathrm{~mm}$ de espessura foram sinterizadas ao ar em forno resistivo (Jung J200) com taxa de aquecimento de $10^{\circ} \mathrm{C} /$ minuto até o patamar da máxima temperatura e com resfria- 
mento por perda térmica do forno até a temperatura ambiente.

Nas amostras dos pós resultantes do processo de sinterização, realizou-se análise de difração de raios X (DRX). As difrações de raios X (Shimadzu XDR 6000) foram realizadas com amostras em pó (200 Mesh), a uma velocidade de $2^{\circ} \theta$ /minuto e em um intervalo de varrimento de 20 a $80^{\circ}$. Os difratogramas de raios $X$ (DRX) foram identificados utilizando-se a base de um programa comercial (Match Phase Identification from Powder Difraction, versão 1.11).

As quantificações das fases por Rietvield (software GSAS - EXPGUI) [25,26] foram realizadas combinando a alumina $\left(\mathrm{Al}_{2} \mathrm{O}_{3}\right)$ na forma de padrão interno (20\%) como condição de correção do deslocamento do ponto zero e quantificação da fase amorfa. $O$ refinamento da cela unitária e os parâmetros de fundo para o grupo espacial Pbnb de estrutura perovskita ortorrômbica só foram realizados após o deslocamento do ponto zero. O fundo foi corrigido utilizando um polinômio Chebyschev do primeiro tipo e os perfis de pico de difração foram ajustados por função pseudo-Voigt. Avaliou-se a qualidade do refinamento por meio dos índices dos mínimos quadrados e índices Rwp, em que o intervalo ideal é entre $2 \%$ e $10 \%$, além de análise gráfica residual [27]. Os índices avaliativos (fatores R) estão listados na seção resultados.

Para determinar as medidas de densidade aparente (hidrostática), as amostras foram fervidas por duas horas em água destilada, resfriadas ao ar, verificada sua massa em balança analítica (AX200 Shimadzu), secas em estufa a $110^{\circ} \mathrm{C} / 4 \mathrm{~h}$, resfriadas ao ar e pesadas novamente para determinar a massa seca (princípio de Arquimedes).

Observou-se a microestrutura das cerâmicas sinterizadas em um microscópio eletrônico de varredura com EDS acoplado (MEV, Shimadzu SS 550). As amostras foram fraturadas manualmente, posteriormente lavadas em ultrassom por 15 minutos e secas em estufa com circulação de ar por 2 horas a $110^{\circ} \mathrm{C}$. Posteriormente, pelo método sputtering, depositou-se uma fina camada de ouro para facilitar a obtenção de imagens (Shimadzu IC 50 Ion Coater).

As amostras foram analisadas pela técnica de espectroscopia de impedância (EIA) em um forno tubular horizontal, com controle de temperatura (controlador Eurotherm 2123), utilizando-se uma ponte de impedâncias Hewlett-Packard 4284A. Os ensaios foram efetuados de $800^{\circ} \mathrm{C}$ a $300{ }^{\circ} \mathrm{C}$, em uma gama de frequências de $1 \mathrm{kHz}$ a $1 \mathrm{MHz}$ em intervalos de $25^{\circ} \mathrm{C}$. A amostra foi colocada em um porta-amostras com a configuração de dois eletrodos. As amostras foram recobertas com uma tinta de platina nas suas superfícies e aquecidas a $900^{\circ} \mathrm{C}$ para sua deposição. Os dados obtidos foram analisados pelo software ISA (Impedance Spectroscopy Analysis) desenvolvido por Dr. João C. C. Abrantes (Instituto Politécnico Viana do Castelo [IPVC] Viana do Castelo, Portugal) e Dr. Jorge R. Frade (Centre for Research in Ceramics and Composite Materials [Ciceco] - Aveiro, Portugal) [28].

\section{RESULTADOS}

A Figura 1 apresenta os difratogramas de raios X (DRX) das matérias-primas utilizadas no desenvolvimento deste trabalho. 


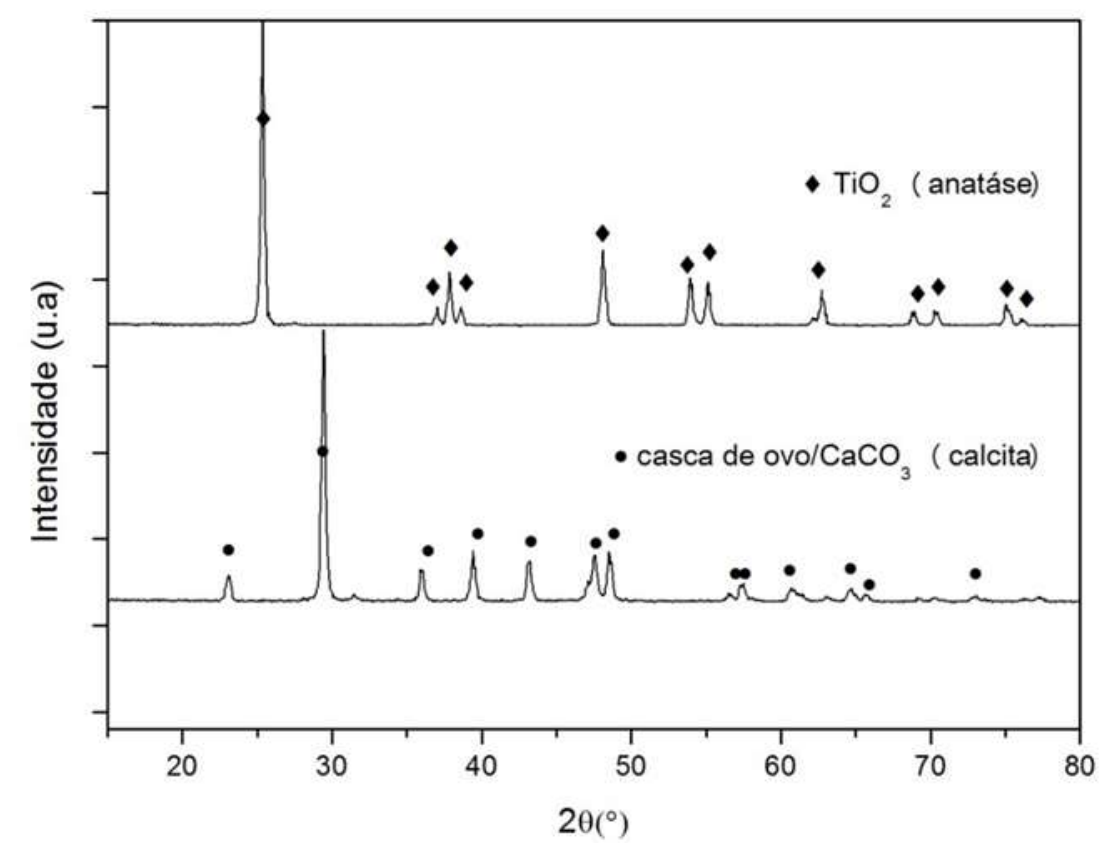

Figura 1: Difratogramas de raios $\mathrm{X}$ das matérias-primas utilizadas. das.

Na Tabela 2 são representados os resultados de fluorescência de raios $\mathrm{X}$ das matérias-primas utiliza-

Tabela 2: Principais compostos mineralógicos presentes nas matérias-primas.

\begin{tabular}{l|l|l}
\hline MATERIAIS & ANATÁSIO (\%) & CALCITA (CASCA DE OVO) (\%) \\
\hline $\mathrm{TiO}_{2}$ & 99 & - \\
\hline $\mathrm{CaCO}_{3}$ & - & 99 \\
\hline Traços de outros elementos químicos & 1 & 1 \\
\hline
\end{tabular}

A fonte de cálcio proveniente da casca de ovo possui alta teor de carbonato de cálcio $(\mathrm{CaCO} 3)$ e valores semelhantes aos encontrados em materiais comerciais e em outros trabalhos realizados [2,3], conforme podemos observar na Tabela 2.

Na Figura 2 são apresentados os difratogramas de raios X das amostras A1, A2 e A3 após a sinterização. 


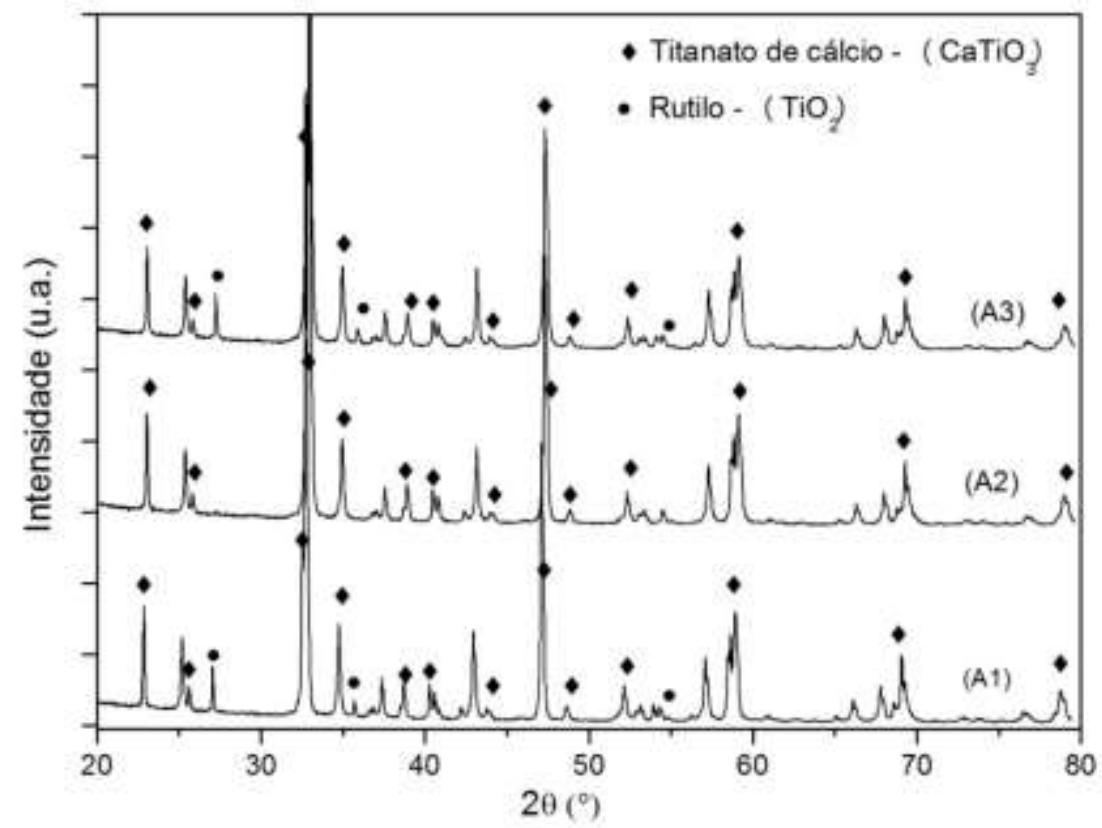

Figura 2: Difratogramas de raios X das composições A1, A2 e A3.

Os principais e mais intensos picos observados nos difratogramas de raios X (Figura 2) são relativos à fase titanato de cálcio $\left(\mathrm{CaTiO}_{3}\right)$, com formação de estrutura ortorrômbica observada em todas as amostras, sendo confirmada como os padrões XRD são combinados com o ICSD n ${ }^{\circ}$ 06-2149 [29].

Na Tabela 3 são apresentadas as porcentagens de fases quantificadas e os fatores que determinam a qualidade do refinamento; para esse tipo de material, pode-se considerar um erro de aproximadamente $\pm 1 \%$ na quantificação de fases. Obteve-se um bom ajuste dos refinamentos com os índices estatísticos ( $\mathrm{x}^{2} \mathrm{e}$ Rpw), sendo os fatores indicativos de qualidade do refinamento realizado aceitáveis (Tabela 3). Com base em trabalhos anteriores [25,30], todos os DRX analisados apresentaram pequenas diferenças de sinal difratométrico em relação àqueles calculados metodologicamente.

Tabela 3: Quantificação das fases formadas nas composições A1, A2 e A3 (com fatores de qualidade dos refinamentos realizados, $\mathrm{R}_{\mathrm{wp}}$ e $\mathrm{x}^{2}$ ).

\begin{tabular}{l|l|l|l|l|l}
\hline AMOSTRA & $\mathrm{CaTiO}_{3}$ & $\mathrm{TiO}_{2}$ & FASE AMORFA & Rwp & $\mathbf{X}^{2}$ \\
\hline $\mathrm{A} 1$ & $69 \pm 1$ & $2 \pm 1$ & $29 \pm 1$ & 6,1 & 2,257 \\
\hline $\mathrm{A} 2$ & $90 \pm 1$ & - & $10 \pm 1$ & 9,65 & 5,249 \\
\hline $\mathrm{A} 3$ & $77 \pm 1$ & $4 \pm 1$ & $19 \pm 1$ & 6,13 & 2,128 \\
\hline
\end{tabular}

Na Tabela 4 são apresentados os valores da densidade aparente, a densidade teórica e a porcentagem de identificação das amostras A1, A2 e A3, respectivamente.

Tabela 4: Densidade aparente das amostras sinterizadas.

\begin{tabular}{|c|c|c|c|}
\hline AMOSTRAS & DENSIDADE APARENTE $\left(\mathrm{g} / \mathrm{cm}^{3}\right)$ & DENSIDADE TEÓRICA $\left(\mathrm{g} / \mathrm{cm}^{3}\right)$ & DENSIFICAÇÃO (\%) \\
\hline A1 & 2,92 & 3,98 & 73,4 \\
\hline A2 & 3,47 & 3,98 & 87,2 \\
\hline A3 & 3,07 & 3,98 & 77,1 \\
\hline
\end{tabular}

Características microestruturais, como tamanho de grãos e porosidade, estão diretamente relacionadas às propriedades elétricas dos materiais. Torna-se relevante analisar suas características por meio das micro- 
grafias apresentadas nas Figuras 3 e 4 .

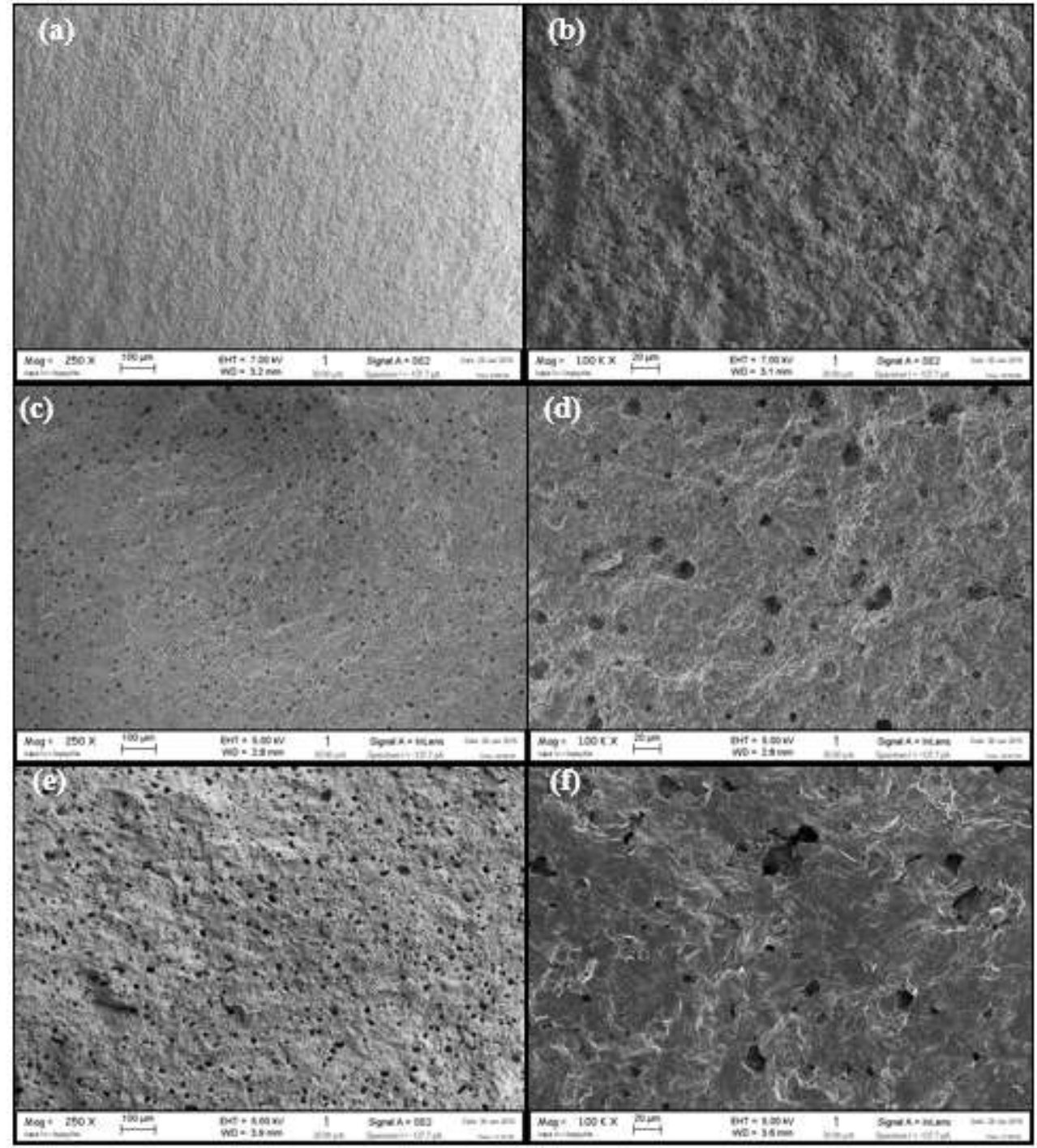

Figura 3: Micrografias das superfícies de fratura das amostras A1 (a, b), A2 (c, d) e A3 (e, f).

Na Figura 4 (a), consta a micrografia da amostra A2, e na Figura 5 (b), o mapa químico realizado via EDS da mesma região.

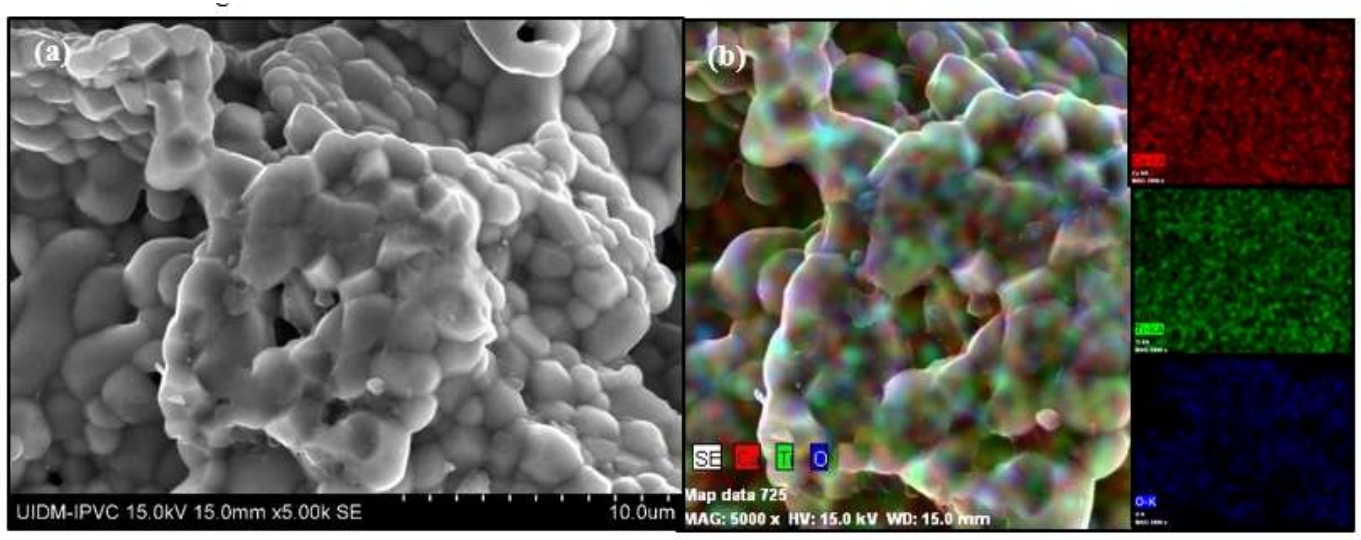

Figura 4: (a) Micrografia do interior de um poro da amostra A2 e (b) mapa químico da amostra A2 obtido via EDS. 
A Figura 5 (a) ilustra as curvas de Arrhenius de composições A1, A2 e A3. Os dados de impedância experimental ajustados da amostra A2 são representados em gráficos de Nyquist a diferentes temperaturas e constam na Figura 6 (b).
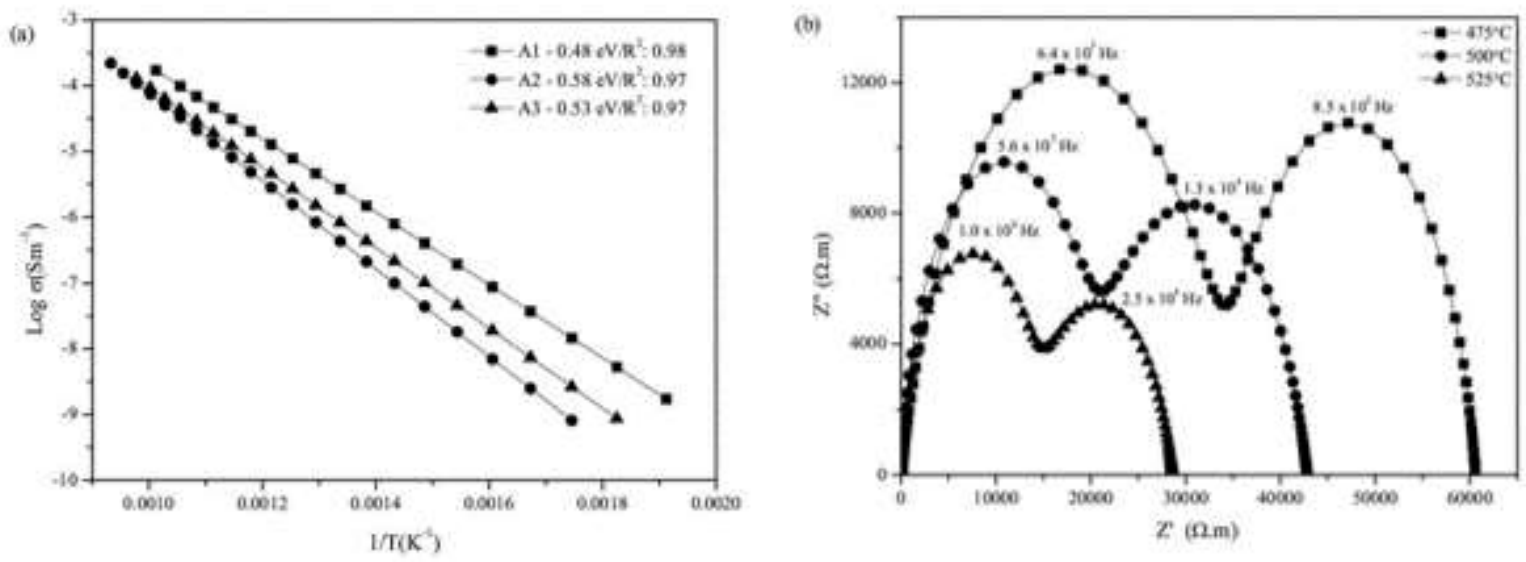

Figura 5: (a) Curvas de Arrhenius de composições A1, A2 e A3, (b) são representados os gráficos de Nyquist da amostra A2 em diferentes temperaturas.

Na Tabela 5 são apresentados os valores de resistência e capacitâncias dos grãos e das fronteiras de grãos da amostra A2.

Tabela 5: Resistência elétrica dos grãos ( $\mathrm{Rg})$, resistência elétrica dos contornos de grãos (Rgb), capacitâncias dos grãos $(\mathrm{Cg})$ e capacitância das fronteiras de grãos $(\mathrm{Cgb})$ da amostra $\mathrm{A} 2$

\begin{tabular}{l|l|l|l|l|l|l}
\hline \multirow{2}{*}{$\begin{array}{l}\text { AMOS- } \\
\text { TRA }\end{array}$} & $\begin{array}{l}\text { TEMPERATURA } \\
\left({ }^{\circ} \mathbf{C}\right)\end{array}$ & $\begin{array}{l}\text { CIRCUITO EQUI- } \\
\text { VALENTE }\end{array}$ & $\begin{array}{l}\mathbf{R}_{\mathbf{g}} \\
(\mathbf{k} \Omega)\end{array}$ & $\begin{array}{l}\mathbf{R}_{\mathbf{g b}} \\
(\mathbf{k} \boldsymbol{\Omega})\end{array}$ & $\begin{array}{l}\mathbf{C}_{\mathbf{g}} \\
(\boldsymbol{\mu} \mathbf{F})\end{array}$ & $\begin{array}{l}\mathbf{C}_{\mathbf{g b}} \\
(\boldsymbol{\mu} \mathbf{F})\end{array}$ \\
\hline \multirow{3}{*}{$\mathrm{A} 2$} & 450 & \multirow{3}{*}{$(\mathrm{RQ})(\mathrm{RQ})$} & $3.72 \times 10^{4}$ & $5.28 \times 10^{4}$ & $1.60 \times 10^{-5}$ & $7.90 \times 10^{-9}$ \\
\cline { 2 - 6 } & 475 & $3.45 \times 10^{4}$ & $2.60 \times 10^{4}$ & $6.70 \times 10^{-11}$ & $3.66 \times 10^{-9}$ \\
\cline { 2 - 6 } & 500 & & $1.98 \times 10^{4}$ & $2.30 \times 10^{4}$ & $1.78 \times 10^{-11}$ & $6.28 \times 10^{-9}$ \\
\hline
\end{tabular}

Conforme se pode observar na Tabela 5, com o aumento da temperatura é evidente a diminuição da resistência elétrica no interior do grão $(\mathrm{Rg})$ e na fronteira do grão $(\mathrm{Rgb})$. Esse fenômeno é comum em materiais semicondutores.

\section{DISCUSSÃO}

E Conforme pode-se observar nos difratogramas de raios X da Figura 1, a fonte de titânio possui somente picos relativos à fase anatásio e a fonte de cálcio possui somente picos relativos à fase calcita. Este resíduo apresenta alta pureza o que facilita na análise de possíveis aplicações. A anátase utilizada é um pigmento branco de titânio comercialmente chamado TIONA, fabricado pela Crystal Brasil S.A. (Camaçari - BA) que utiliza o processo sulfato na obtenção de anatásio $\left(\mathrm{TiO}_{2}\right)$, em que o ácido sulfúrico extrai a anatásio dos minérios rutilo e ilmenita com elevadas porcentagens de $\mathrm{TiO}_{2}$, o produto final comercializado possui alta pureza $(99 \%)$.

Observam-se também picos de alumina $\left(\mathrm{Al}_{2} \mathrm{O}_{3}\right)$ provenientes do padrão adicionado para realizar refinamento pelo método Rietveld (picos não identificados). Apesar de os difratogramas apresentarem um perfil muito semelhante, pode-se observar uma diferença no perfil da composição A2 em relação às demais (A1 e A3), por volta de $27^{\circ}$ e $36^{\circ}$. Picos característicos da fase rutilo podem ser observados nas amostras A1 e A3; já a composição $\mathrm{A} 2$, sinterizada a $1.200^{\circ} \mathrm{C}$ por 13 horas, não apresenta a fase rutilo. Tal fato torna-se relevante quando considerado o longo processamento térmico ao qual foi submetida a amostra A2, sendo toda o anatásio consumida para a formação de titanato de cálcio $\left(\mathrm{CaTiO}_{3}\right)$ e fase amorfa, conforme foi confirmado nas quantificações de fase que serão discutidas a seguir.

Conforme se pode verificar na Tabela 3, a maior quantidade de titanato de cálcio $\left(\mathrm{CaTiO}_{3}\right)$ formado 
ocorreu na composição A2. Foi com essa composição e condição de processamento $\left(1.200^{\circ} \mathrm{C}\right.$ por 13 horas de patamar) que se alcançou a maior densificação dos corpos sinterizados. Nas amostras A1 e A3, pode-se observar anatásio remanescente e elevadas quantidades de fase amorfa. Temperaturas mais baixas de sinterização, em que a amostra A1 foi calcinada, apresentam a maior quantidade de fase amorfa e a menor quantidade de titanato de cálcio $\left(\mathrm{CaTiO}_{3}\right)$ formado.

Os resultados apresentados na Tabela 3 apresentam considerável quantidade de fase amorfa em todas as amostras, sendo a amostra A1 a que apresenta a maior quantidade (29\%). O estudo de estruturas de materiais amorfos é muito interessante quando comparado com o de estruturas cristalinas, porém há grande dificuldade em termos de estudo na ordem estrutural de materiais amorfos, que é o estudo de ordem a média distância [31,32].

O processo de densificação das amostras está mais relacionado ao tempo na máxima temperatura de sinterização do que ao valor da temperatura utilizada, conforme podemos observar na Tabela 4. As amostras A1 e A3 obtiveram valores de densidade aparente próximos e sofreram tempos de permanência mais curtos, de três e duas horas respectivamente. O valor correspondente ao de máxima densificação alcançada $(3,47$ $\mathrm{g} / \mathrm{cm} 2$ ) foi obtido na amostra A2 com 13 horas de sinterização. Esse elevado tempo de sinterização (13 horas) ocorreu por um problema no forno, não sendo intencional.

Na Figura 4 (a), (c) e (e), pode-se verificar a formação de porosidades fechada e aberta com poros arredondados, provenientes provavelmente da formação de gases durante o processo de sinterização. Maior quantidade de poros e com tamanhos maiores é observada na composição A3. Poros com menor dimensão são observados na amostra A1. O processo de fratura das amostras não permitiu observar o tamanho dos grãos, o que seria interessante para avaliar características físicas importantes.

Na Figura 4 (a), pode-se observar grãos com um tamanho aproximado de $2 \mu \mathrm{m}$. Essa imagem foi obtida do interior de um poro da amostra A2. Nessa mesma região foi realizada uma microanálise química via EDS (Figura 4 [b]). No mapa químico, pode-se observar alta homogeneidade dos componentes químicos. Mapas químicos individuais são mostrados ao lado da Figura 4 (b), onde a cor vermelha corresponde ao elemento químico cálcio ( $\mathrm{Ca}$ ), a cor verde, ao elemento químico titânio (Ti), e a cor azul, ao elemento químico oxigênio (O). Todos os elementos químicos estão distribuídos de maneira uniforme, formando o titanato de cálcio $\left(\mathrm{CaTiO}_{3}\right)$ de maneira homogênea.

A espectroscopia de impedância é uma técnica utilizada para caracterizar as propriedades elétricas dos materiais, podendo ser qualitativamente caracterizadas pela demonstração de diferentes regiões eletricamente ativas e quantitativamente pela medição das suas propriedades elétricas individuais por meio da análise de dados apropriados[33]. A condutividade de Dc pode ser calculada com base nas dimensões da amostra, enquanto a resistência é obtida a partir da intercepção no eixo real. A energia de ativação, Edc, foi calculada pelo método dos mínimos quadrados (Figura 5 [a]).

O gráfico de Arrhenius indica grãos de natureza semicondutora na faixa de 0,58 eV a 0,48 eV. As dispersões maiores entre as curvas estão representadas em baixas temperaturas. Todas as curvas apresentaram elevado coeficiente de correlação (R2), o que indica que o modelo utilizado é apropriado aos dados obtidos. A amostra A2 apresenta o maior valor de energia de ativação $(0,58 \mathrm{eV})$ e também possui a maior quantidade de titanato de cálcio $\left(\mathrm{CaTiO}_{3}\right)$ com $90 \%$, conforme indica a Tabela 4. Já o menor valor de energia de ativação foi encontrado na amostra A1 $(0,48 \mathrm{eV})$, que possui a maior quantidade de fase amorfa (29\%), conforme indica a Tabela 4. A amostra A3 possui um valor de energia de ativação intermediário $(0,53 \mathrm{eV})$, sendo essa amostra sinterizada a $1.300^{\circ} \mathrm{C}$.

As amostras do presente estudo evidenciaram a contribuição que as diferentes regiões podem obter das simulações de dados experimentais realizadas em elementos de circuitos equivalentes, resistências (R) e capacitores (C). O circuito desenvolvido para a amostra aqui estudada consistiu em dois subcircuitos: um representou o efeito do interior do grão, enquanto o outro correspondeu às distribuições do contorno do grão. Cada subcircuito consiste em uma combinação entre um resistor e um capacitor. Sejam ( $\operatorname{Rg}, \operatorname{Rgb})$ e $(\mathrm{Cg}$, $\mathrm{Cgb})$ as resistências e capacitâncias dos grãos e das fronteiras dos grãos, respectivamente.

A Figura 5 (b) mostra os espectros de impedância na amostra A2, o que indica um componente resistivo/capacitivo bem definido. Selecionou-se a amostra A2 pelo alto valor de densidade aparente e pela alta quantidade de titanato de cálcio quantificadas pelo método Rietveld (Tabela 3). O valor máximo da parte imaginária da impedância na amostra é encontrado na frequência de 6,4 x $105 \mathrm{~Hz}$. Em geral, semicírculos associados ao grão e ao contorno de grão começam a se definir quando suas frequências de relaxação diferem de duas ordens de grandezas. Mais de um semicírculo traçado no presente estudo mostrou muitas regiões eletricamente ativas que correspondem aos mecanismos elétricos no interior e no contorno do grão. As parcelas são semicirculares e sua interceptação no eixo real deslocou-se em direção à origem, à medida que a tem- 
peratura aumentava. O semicírculo relacionado com as reações que ocorrem na interface eletrólito/eletrodo não é observado em razão dos efeitos de relaxação em baixas frequências.

A porosidade aumenta a resistividade, o que é observado de duas formas nos diagramas de impedância: por meio de aumento no diâmetro do semicírculo correspondente ou como um semicírculo em frequência intermediária. O primeiro tipo de bloqueio foi observado preferencialmente em relação à porosidade intragranular[34], enquanto o segundo se relaciona à porosidade intergranular. Trincas e microtrincas também podem gerar um bloqueio aos portadores de carga [35]. Assim, a amostra A3, sinterizada por duas horas e contendo porosidade intragranular, deve apresentar maior resistividade elétrica comparada a uma amostra com igual composição, mas que não apresente porosidade no interior dos grãos, sem, entretanto, dar origem a um semicírculo adicional no diagrama de impedância. sta é a seção adequada do artigo para se adicionar conteúdo científico, pela análise dos resultados apresentados com o devido suporte da literatura e pelo modelamento e simulação de comportamentos e condições específicas.

\section{CONCLUSÕES}

Com base nos resultados obtidos, pode-se concluir que ocorreu a formação de titanato de cálcio $\left(\mathrm{CaTiO}_{3}\right)$ com estrutura perovskita nas composições analisadas, utilizando-se como fonte alternativa de cálcio o resíduo da casca de ovo de consumo doméstico. Esse resíduo apresentou alta pureza e as condições de sinterização mostraram que um maior tempo de calcinação promoveu aumento na quantidade de titanato de cálcio (Ca$\mathrm{TiO}_{3}$ ) formado e maior densificação das amostras. As propriedades elétricas são promissoras, podendo ser analisadas possíveis aplicações como sensores de temperatura do tipo termistores NTC, uma maior quantidade de titanato de cálcio proporcionou um maior valor na energia de ativação; em contrapartida uma maior quantidade de fase amorfa proporcionou um menor valor na energia de ativação.

\section{AGRADECIMENTOS}

Agradecimento especial ao professor Dr. João C. C. Abrantes (Instituto Politécnico Viana do Castelo [IPVC] - Viana do Castelo, Portugal) pela análise de espectroscopia de impedância, e aos recursos disponibilizados pelo $\mathrm{CNPq} / \mathrm{CAPES}$.

\section{BIBLIOGRAFIA}

[1] Lei $\mathrm{N}^{\circ} 12.305$, de 2 de agosto de 2010, http://www.planalto.gov.br/ccivil_03/_ato20072010/2010/lei/112305.htm, acessado em dezembro de 2016.

[2] GOMES, L. C., Di LLELlO, B. C., CAMPOS, J. B., et al., "Síntese e caracterização de fosfatos de cálcio a partir da casca de ovo de galinha", Cerâmica, v. 58, n 348, pp. 448- 452, Dez. 2012.

[3] FREIRE M. N., HOLANDA J. N. F., "Caracterização de resíduo de casca de ovo visando seu aproveitamento em revestimento cerâmico poroso", Cerâmica, v. 52, n. 324, pp. 240-244, Dez. 2006.

[4] MAGALHÃES M. C., RIBEIRO A., QUINA M., et al., "Tratamento e valorização agrí́cola da casca de ovo", Revista de Ciências Agrárias, v.34, n. 2, pp. 191-204, Jul. 2011.

[5] OLIVEIRA T. C., "Caracterização elétrica e dielétrica de cerâmicas de titanato de bário e cálcio", Tese de M.Sc., NPGFI/UFS, São Cristóvão, SE, Brasil, 2011.

[6] KENNEDY B. J., HOWARD C. J., CHAKOUMAKOS B. C., "Phase transitions in perovskite at elevated temperatures - a powder neutron diffraction study", Journal of Physics Condensed Matter. v. 11, n. 6, pp. 1479-1488, Out. 1999.

[7] GONÇALVES R. F., CARRENO N. L. V., ESCOTE M. T., et al., "Fotoluminescência e Adsorção de $\mathrm{CO}_{2}$ em Nanopartículas de $\mathrm{CaTiO}_{3}$ Dopadas com Lantânio", Química Nova, v. 27, n,6, pp, 862-865, Ago. 2004.

[8] MI G. M., MURAKAMI Y., SHINDO D., et al., "Microstructural investigation of $\mathrm{CaTiO}_{3}$ formed mechanochemically by dry grinding of a $\mathrm{CaO}-\mathrm{TiO}_{2}$ mixture", Powder Technology, v. 104, n. 1, pp. 75-79, Ago. 1999.

[9] MOULSONN A. J.; HERBERT J. M., Electroceramics: Materials, Properties and Applications, 2 ed., New York, John Wiley \& Sons, 2003.

[10] KIM J. W., YOON D. C., JEON M. S., et al., "Degradation behaviors and failure analysis of Ni-BaTiO base-metal electrode multilayer ceramic capacitors under highly accelerated life test", Current Applied Physics, v. 10, n. 5, pp. 1297-1301, Set. 2010. 
[11] BELL A. J. "Ferroelectrics: The role of ceramic science and engineering", Journal of the European Ceramic Society, v. 28, n. 7, pp. 1307-1317, Jan. 2008.

[12] LEMANOV V. V., SOTNIKOV A.V., SMIRNOVA E. P., et al., "Perovskite $\mathrm{CaTiO}_{3}$ as an incipient ferroelectric", Solid State Communications, v. 110, n.11, pp. 611-614, Abr. 1999.

[13] HAO J., SI W., Xi X. X., et al.,; "Dielectric properties of pulsed-laser-deposited calcium titanate thin films", Applied Physics Letter, v. 76, n. 21, pp. 3100-3102, Mai. 2000.

[14] WEBSTER T. J., ERGUN, C., DOREMUS, R. H., et al.,"Increased osteoblast adhesion on titanium coated hydroxylapatite that forms $\mathrm{CaTiO}_{3}$ "Journal of Biomedical Materials Research Part A, v. 67, n. 3, p. 975-980, 2003.

[15] MI G. M., MURAKAMI Y., SHINDO D., et al., "Microstructural investigation of $\mathrm{CaTiO}_{3}$ formed mechanochemically by dry grinding of a $\mathrm{CaO}-\mathrm{TiO}_{2}$ mixture", Powder Technology, v. 104, n. 1, pp. 75-79, Jul. 1999.

[16] FIGUEIREDO, A. T., LONGO, V. M., LAZARO, S., et al., "Blue-green and red photoluminescence in $\mathrm{CaTiO}_{3}:$ Sm", Journal of Luminescence, v. 126, n. 2, pp. 403-407, Nov. 2007.

[17] FIGUEIREDO, A. T., LAZARO, S., LONGO, V. M., et al., "Correlation among Order-Disorder, Electronic Levels, and Photoluminescence in Amorphous CT:Sm", Chemistry of Materials, v. 18, n. 12, pp. 29042911, Mai. 2006.

[18] GEORGE W. L., GRACE, R. E. "Formation of point defects in calcium titanate", Journal Physics Chemistry Solids, v. 30, n. 4, pp. 881-887, Set. 1969.

[19] THOMAS D. G., LANDER J. J., "Hydrogen as a Donor in Zinc Oxide", Journal Chemical Physics, v. 25 , n. 6, pp. 1136-1142, Fev. 1956.

[20] WALTERS L. C., GRACE R. E., "Diffusion of point defects in strontium titanate", Journal Physics Chemistry Solids, v. 28, n. 2, pp. 245-248, Fev. 1967.

[21] IWAHARA H., ESAKA T., MANGAHARA T., "Mixed conduction and oxygen permeation in the substituted oxides for $\mathrm{CaTiO}_{3}$ ”, Journal Applied Electrochemical, v. 18, n. 2, pp. 173-177, Mar. 1988.

[22] TAKAHASHI T., IWAHARA H., ICHIMURA T., "Ionic Conduction in Solid Solutions of CaTi ${ }_{x} \mathrm{Al}_{\mathrm{x}} \mathrm{O}_{3-\alpha} 0$,a ", Chemical Abstracts, v. 37, n. 3, pp. 857-862, 1969.

[23] MUCCILlO E. N. S., PORFIRIO T. C., TADOKORO S. K., et. al., "Propriedades físicas do óxido de cério preparado por técnica de solução e a condutividade elétrica em função da pressão parcial de oxigênio em cerâmicas sinterizadas", Cerâmica, v. 51, n. 318, pp. 157-162, Jun. 2005.

[24] MUCCILLO E. N. S., BUISSA NETO R. C., TAKADORO S. K., et. al., "Preparação, sinterização e espectroscopia de impedância da zircônia parcialmente estabilizada com cálcia" Cerâmica, v. 52, n. 323, pp. 207-214, Set. 2006.

[25] LARSON A. C., DREELE R. B. B., GSAS, https://permalink.lanl.gov/object/tr?what=info:lanlrepo/lareport/LA-UR-86-0748_REV, acessado em outubro de 2019.Los Alamos National Laboratory Report LAUR (2000) 86.

[26] TOBY B. H., "EXPGUI, a graphical user interface for GSAS", Journal Applied Crystallography, v. 34, n. 2, pp. 210-213, Fev. 2001

[27] TOBY B. H., "R factors in Rietveld analysis: How good is good enough?", Powder Diffraction, v. 21, n.1, pp. 64-70, Mar. 2006.

[28] ABRANTES J. C. C., LABRINCHA J. A., FRADE J. R., "An alternative representation of impedance spectra of ceramics", Materials Research Bulletin, v. 35, n. 5, pp. 727-740, Mar. 2000.

[29] ICSD (Inorganic Crystal Structure Database), JCPDS Card No. 06 - 2149.

[30] SINDHU M., AHLAWAT N., SANGHI S., et. al., "Rietveld refinement and impedance spectroscopy of calcium titanate", Current Applied Physics, v. 12, n. 6, pp. 1429-1435, Nov. 2012.

[31] HUFNAGEL T. C., "Finding order in disorder", Nature Materials, v.3, n.10, pp. 666-667, Out. 2004.

[32] CHEN G. L., LIU X. J., HUI X. D., et. al., "Molecular dynamic simulations and atomic structures of amorphous materials", Applied Physics Letter, v. 88, n. 20, pp. 203115, Mai. 2006.

[33] IDAULT O., GOUX P., KCHIKECH M., et. al., "Space-charge relaxation in perovskites", Physics Review $B$, v. 49, n. 12, pp 7868-7873, Mar. 1994. 
[34] MCDONALD J. R., Impedance Spectroscopy-emphasizing solid materials and systems, 1 ed., New York, John Wiley \& Sons, 1987.

[35] LEACH C., "Microcrack observations using a.c. impedance spectroscopy, Journal Materials Science Letters, v.11, n. 5, pp. 306-307, Jan. 1992.

\section{ORCID}

Guilherme Gralik

https://orcid.org/0000-0002-9242-6679

Giovany Biava

https://orcid.org/0000-0002-8382-101X 